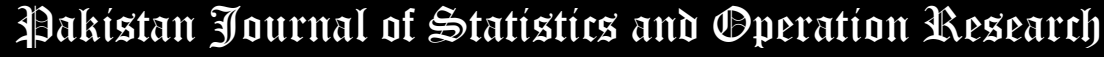

\section{A novel four-parameter log-logistic model: mathematical properties and applications to breaking stress, survival times and leukemia data}

\author{
Wahid A. M. Shehata ${ }^{1 *}$, Murtadha Mansour Abdullah ${ }^{2}$ \\ Mohamed K. A. Refaie ${ }^{3}$
}

*Corresponding Author

${ }^{1}$ Department of Mathematics, Statistics and Insurance, Faculty of Business, Ain Shams University, Egypt. wahid75maher@yahoo.com

${ }^{2}$ University of Wasit. College of Administration and Economics, Iraq. mabdullah@uowasit.edu.iq

${ }^{3}$ Agami High Institute of Administrative Sciences, Alexandria, Egypt. refaie_top@yahoo.com

\begin{abstract}
In this paper, we introduce a new continuous log-logistic extension. Several of its properties are established. A numerical analysis for skewness and kurtosis is presented. The new failure rate can be "bathtub or $\mathbf{U}$ shaped", "increasing", "decreasing-constant", "J shaped", "constant" and "decreasing". Many bivariate and Multivariate type distributions are derived using the Clayton Copula and the Morgenstern family. To assess of the finite sample behavior of the estimators, we performed a graphical simulation. Some useful applications are considered for supporting the new model.
\end{abstract}

Key Words: Burr XII Distribution; Log-Logistic Model; Maximum Likelihood; Leukemia Data; Modeling; Simulation.

Mathematical Subject Classification: 62N01; 62N02; 62E10.

\section{Introduction and motivation}

A continues random variable (RV) $Y$ is said to has the log-logistic (LL) model if its survival/reliability function (SF) be written as

$$
\bar{S}_{b}(y)=1-S_{b}(y)=\left(1+y^{b}\right)^{-1},
$$

where $b>0$ is a shape parameter and $S_{b}(y)$ refer to the cumulative distribution functions (CDF) of the LL model. The density function (PDF) due to (1) is given as

$$
s_{b}(y)=b y^{b-1}\left(1+y^{b}\right)^{-2} .
$$

The CDF and PDF in (1) and (2) is a sub-model from the Burr model from the type-XII (BUXII) model (Burr (1942, 1968 and 1973), Tadikamalla (1980) and Rodriguez (1977)). Due to Yousof et al. (2018), the CDF of the new Weibull Generalized log-logistic (WG-LL) is defined as

$$
F(y)=1-e^{-a\left[\left(1+y^{b}\right)^{\beta}-1\right]^{\alpha}},
$$

where $y>0, \alpha, \beta, a>0$ and its corresponding PDF is given by

$$
f(y)=\alpha \beta a b y^{b-1}\left(1+y^{b}\right)^{\beta-1}\left[\left(1+y^{b}\right)^{\beta}-1\right]^{\alpha-1} e^{-a\left[\left(1+y^{b}\right)^{\beta}-1\right]^{\alpha}} .
$$

Figure 1 below gives some graphical results for the novel PDF and its corresponding HRF for the WG-LL model. Due to Fig. 1(left chart) it is seen that the novel PDF of the WG-LL model can be monotonical and unimodal PDF, symmetric PDF or negative skewed PDF. From Figure 1(right chart) the HRF can be "bathtub" $(\alpha=0.85, \beta=$ 
$0.5, a=0.001, b=0.55) \quad$ or $\quad$ "increasing (monotone-HRF)" $(\alpha=0.95, \beta=7, a=0.0001, b=0.55) \quad$ or "decreasing-constant" ( $\alpha=0.85, \beta=3, a=0.05$, $b=0.5$ ) or "J-shaped" ( $\alpha=2, \beta=5, a=0.01, b=1.5)$ or "constant" ( $\alpha=1, \beta=1, a=1, b=1)$ or "decreasing (monotone-HRF)" $(\alpha=1, \beta=0.5, b=a=1)$.
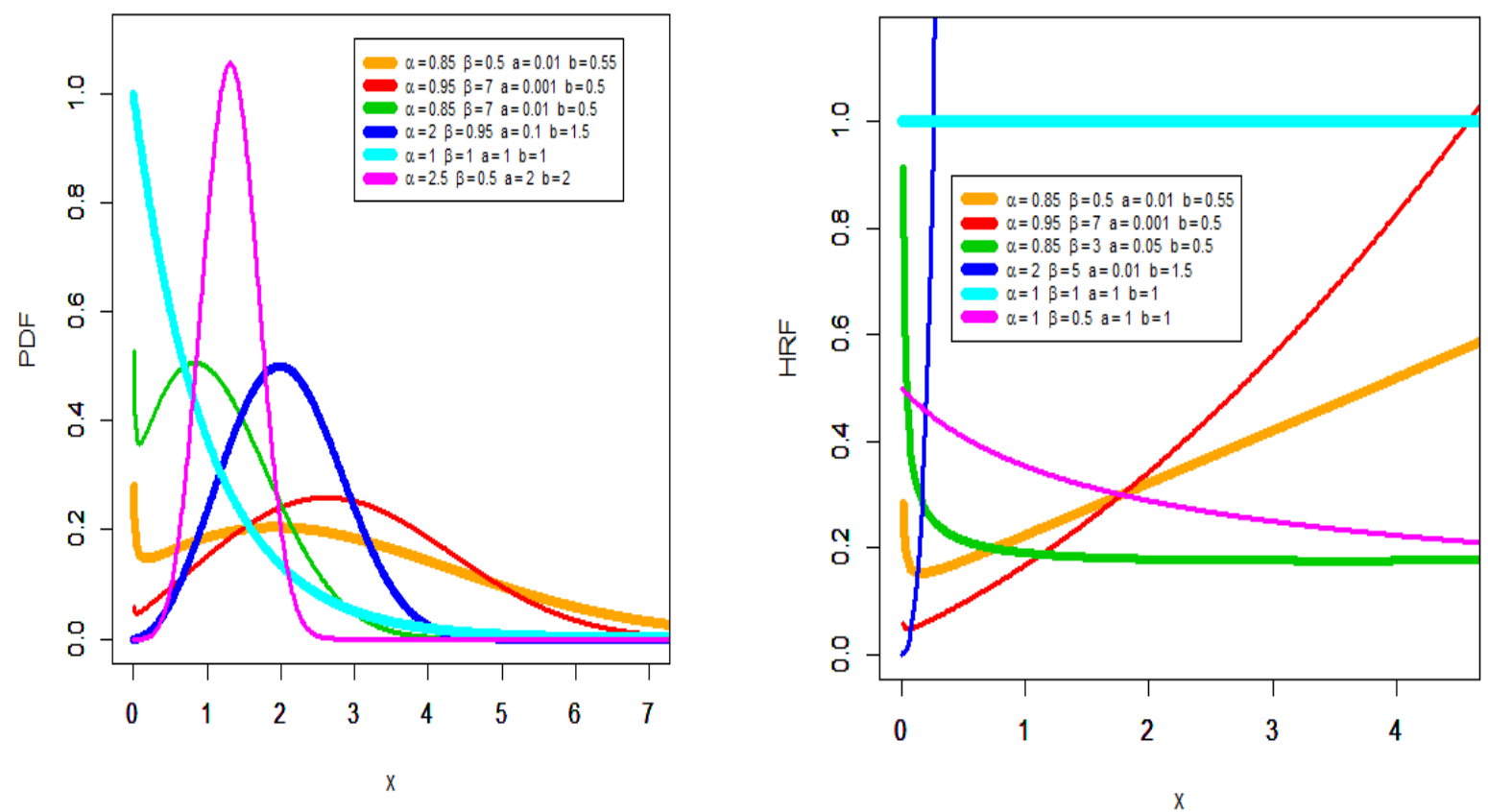

Figure 1: graphical charts of the novel PDF and it corresponding HRF.

Due to Yousof et al. (2018), the new PDF in (4) can be re-expressed as

$$
f(y)=\sum_{j_{4}=0}^{\infty} C_{\left[j_{4}\right]} S_{\left[\ell,\left(1+j_{4}\right)\right]}(y),
$$

where $s_{\left[b,\left(1+j_{4}\right)\right]}(y)=\left(1+j_{4}\right) b y^{b-1}\left(1+y^{b}\right)^{-2-j_{4}}$ is the LL PDF with $b$ and $\left(1+j_{4}\right)$ where

$$
C_{\left[j_{4}\right]}=\sum_{j_{1}, j_{2}, j_{3}=0}^{\infty}(-1)^{1+j_{1}+j_{2}+j_{3}+j_{4}} \frac{1}{j_{1} ! j_{4} !}\left(\begin{array}{c}
j_{1} \alpha \\
j_{2}
\end{array}\right)\left(\begin{array}{c}
\beta\left(j_{2}-j_{1} \alpha\right) \\
j_{3}
\end{array}\right)\left(\begin{array}{c}
1+j_{3} \\
j_{4}
\end{array}\right),
$$

Analogously, the new CDF (3) can be re-expressed as

where

$$
F(y)=\sum_{j_{4}=0}^{\infty} C_{\left[j_{4}\right]} S_{\left[b,\left(1+j_{4}\right)\right]}(y)
$$

$$
S_{\left[b,\left(1+j_{4}\right)\right]}(y)=-\left(1+y^{b}\right)^{-1-j_{4}}+1
$$

represents the CDF of the well-known LL model with parameters $b$ and $\left(1+j_{4}\right)$. This work can be motivated via the following applied justifications:

$\boldsymbol{i}$. The new model in its novel pattern will be useful in mathematical modeling of the engineering real-life datasets such as the "monotonically-increasing HRF" engineering breaking stress real-life dataset.

ii. The novel model in its novel version can be used for statistical modeling of the reliability real-life datasets such as the "monotonically-increasing HRF" reliability real-life dataset.

iii. The new current version of the log-logistic version can be used in modeling the medical real-life datasets such as the "U- HRF" medical real-life dataset. 


\section{Properties}

\section{Moments and generating function}

The $\mathrm{m}^{\text {th }}$ ordinary moment of $Y$ is given by

Then, we obtain

$$
\mu_{m, Y}^{\prime}=E\left(Y^{m}\right)=\int_{-\infty}^{\infty} f(y) y^{m} d y .
$$

where

$$
\mu_{m, Y}^{\prime}=\left.\sum_{j_{4}=0}^{\infty} C_{\left[j_{4}\right]}\left(1+j_{4}\right) B\left(1+\frac{m}{b},\left(1+j_{4}\right)-\frac{m}{b}\right)\right|_{\left(m<\left(1+j_{4}\right) b\right)},
$$

$$
B\left(d_{1}, d_{2}\right)=\int_{0}^{\infty} t^{d_{1}-1}(1+t)^{-\left(d_{1}+d_{2}\right)} d t
$$

is the second type of beta function. By fixing $m=1$ in $\mu_{m, Y}^{\prime}$, we obtain the mean of the model. The $\mathrm{m}^{\text {th }}$ incomplete moment $\left(I_{n}(t)\right)$ of $Y$ can be expressed from (3) as

where

$$
I_{m, Y}(t)=\int_{-\infty}^{t} y^{m} f(y) d y=\left.\sum_{j_{4}=0}^{\infty} C_{\left[j_{4}\right]}\left(1+j_{4}\right) B\left(t^{b} ; 1+\frac{m}{b},\left(1+j_{4}\right)-\frac{m}{b}\right)\right|_{\left(m<\left(1+j_{4}\right) b\right)},
$$

$$
B\left(q ; a_{1}, a_{2}\right)=\int_{0}^{q} t^{a_{1}-1}(1+t)^{-\left(a_{1}+a_{2}\right)} d t
$$

Is the second type of the incomplete beta function. The moment generating function (mgf) $M_{Y}(t)=E(\exp (t Y))$ of $Y$ can be derived from (3) as

$$
M_{Y}(t)=\left.\sum_{j_{4}, n=0}^{\infty} \frac{t^{n}}{n !} C_{\left[j_{4}\right]}\left(1+j_{4}\right) B\left(1+\frac{n}{b},\left(1+j_{4}\right)-\frac{n}{b}\right)\right|_{\left(n<\left(1+j_{4}\right) b\right)},
$$

\section{Probability weighted moments (PWMs)}

The $(\mathrm{m}, \mathrm{r})^{\text {th }} \mathrm{PWM}$ of $Y$ can be expressed as

where

$$
p_{m, r, Y}=\left.\sum_{r=0}^{\infty} C_{[r]}(1+r) B\left(\frac{m}{b}+1,(1+r)-\frac{m}{b}\right)\right|_{(m<(1+r) b)},
$$

$$
\begin{aligned}
& C_{[r]}=\alpha \beta a \sum_{i, j_{1}, j_{2}, j_{3}=0}^{\infty}(-1)^{i+j_{1}+j_{2}+j_{3}} \frac{(1+i)^{j_{1}}}{\Gamma(1+r) \Gamma\left(1+j_{1}\right) !}(r)_{j_{3}} \\
& \times\left(\begin{array}{c}
1+j_{3} \\
r
\end{array}\right)\left(\begin{array}{c}
\left(1+j_{1}\right) \alpha-1 \\
j_{2}
\end{array}\right)\left(\begin{array}{c}
\beta\left[-\left(1+j_{1}\right) \alpha+j_{2}\right]-1 \\
j_{3}
\end{array}\right),
\end{aligned}
$$

and $\left(c_{1}\right)_{c_{2}}=c_{1}\left(c_{1}-1\right) \ldots\left(1+c_{1}-c_{2}\right)$ is the common factorial for the descending king and $c_{2}$ should be integer and also positive.

\section{Reversed residual life Moment (MRRL) function}

The $m^{\text {th }}$ MRRL, say

Then, we have

$$
A_{m, Y}(t)=E\left[\left.(t-y)^{m}\right|_{(y \leq t, t>0, m=1,2, \ldots)}\right]
$$

Then, the $m^{\text {th }}$ MRRL of $Y$ becomes

$$
A_{m}(t)=F^{-1}(t) \int_{0}^{t}(t-y)^{m} d F(y) .
$$

Where

$$
A_{m, Y}(t)=F^{-1}(t) \sum_{r=0}^{\infty} C_{\left[j_{4}\right]}^{(\omega)}\left(1+j_{4}\right) B\left(t^{b} ;\left(1+j_{4}\right)-\frac{m}{b}, 1+\frac{m}{b}\right),
$$

$$
C_{\left[j_{4}\right]}^{(\omega)}=C_{\left[j_{4}\right]} \sum_{r=0}^{m}(-1)^{r}\left(\begin{array}{c}
m \\
r
\end{array}\right) t^{m-r} .
$$




\section{Checking flexibility numerically}

In this section, the impacts of the parameters on the model mean $\left(\mu_{1}^{\prime}\right)$, variance of the model $(\mathrm{V}(X))$, skewness of the model $(\mathrm{S}(X))$ and kurtosis of the model $(\mathrm{K}(X))$ are given below in Table 1. The impacts of $b$ for the standard LL model on the $\mu_{1}^{\prime}, \mathrm{V}(X), \mathrm{S}(X)$ and $\mathrm{K}(X)$ are provided in Table 2.

For the new WG-LL model, $\mathrm{S}(x) \in(-1.08107,16.7425)$. However, for the LL model, $\mathrm{S}(X) \in(0.0872,2.4853)$. For the novel WG-LL model, $\mathrm{K}(x) \in(3.2451,702.5)$. However, for the LL model, $\mathrm{K}(X) \in(3.7409,29.5562)$.

Table 1: $\mu_{1}{ }^{\prime}, \mathrm{V}(\mathrm{X}), \mathrm{S}(\mathrm{x}), \mathrm{K}(\mathrm{X})$ for the WG-LL model.

\begin{tabular}{|c|c|c|c|c|c|c|c|}
\hline$\alpha$ & $\beta$ & $\mathrm{a}$ & $\mathrm{b}$ & $\mu_{1}{ }^{\prime}$ & $\mathrm{V}(\mathrm{X})$ & $\mathrm{S}(\mathrm{X})$ & $\mathrm{K}(\mathrm{X})$ \\
\hline 0.35 & 1 & 1 & 1 & 5.029144 & 399.0459 & 16.74246 & 702.4982 \\
\hline 0.5 & & & & 2 & 20 & 6.618761 & 87.72 \\
\hline 1 & & & & 1 & 1 & 2 & 9 \\
\hline 2 & & & & 0.8862269 & 0.2146018 & 0.6311107 & 3.245089 \\
\hline 10 & & & & 0.9513508 & 0.01310046 & -0.6376371 & 3.570166 \\
\hline 35 & & & & 0.984295 & 0.001249773 & -0.978317 & 4.684532 \\
\hline 50 & & & & 0.9888442 & 0.0006253426 & -1.024853 & 4.877788 \\
\hline 75 & & & & 0.9924775 & 0.0002825923 & -1.062093 & 5.040069 \\
\hline 100 & & & & 0.9943259 & 0.0001603049 & -1.081074 & 5.125489 \\
\hline \multirow[t]{7}{*}{5} & 0.1 & 3 & 2 & 17.25458 & 63.62621 & 0.749758 & 3.620892 \\
\hline & 0.5 & & & 1.414735 & 0.04437565 & -0.4251616 & 3.159187 \\
\hline & 1 & & & 0.8523708 & 0.01051628 & -0.6376371 & 3.570166 \\
\hline & 2.5 & & & 0.4929942 & 0.00267863 & -0.7801194 & 3.933523 \\
\hline & 5 & & & 0.3387453 & 0.001151769 & -0.8305135 & 4.079192 \\
\hline & 7.5 & & & 0.2739872 & 0.000730451 & -0.8549788 & 4.244319 \\
\hline & 10 & & & 0.2361687 & 0.0005340107 & -0.8562963 & 4.157227 \\
\hline \multirow[t]{13}{*}{2.5} & 1.5 & 0.00001 & 3 & 2.618206 & 0.0872134 & -0.7195030 & 3.804983 \\
\hline & & 0.0001 & & 2.108410 & 0.0601449 & -0.7321432 & 3.837628 \\
\hline & & 0.001 & & 1.684376 & 0.0419942 & -0.7383334 & 3.839446 \\
\hline & & 0.01 & & 1.329646 & 0.0294111 & -0.7251535 & 3.773603 \\
\hline & & 0.1 & & 1.033039 & 0.0201868 & -0.6837104 & 3.636145 \\
\hline & & 0.5 & & 0.856814 & 0.0150825 & -0.6422962 & 3.525361 \\
\hline & & 1 & & 0.788435 & 0.0131753 & -0.6236186 & 3.481136 \\
\hline & & 10 & & 0.592367 & 0.0080480 & -0.5684401 & 3.367608 \\
\hline & & 50 & & 0.481838 & 0.0055071 & -0.5412732 & 3.320269 \\
\hline & & 200 & & 0.402187 & 0.0039072 & -0.5256268 & 3.295386 \\
\hline & & 500 & & 0.356572 & 0.0030960 & -0.5184591 & 3.284576 \\
\hline & & 5000 & & 0.262971 & 0.0017034 & -0.5079508 & 3.269382 \\
\hline & & 10000 & & 0.239855 & 0.0014198 & -0.5061509 & 3.266805 \\
\hline \multirow[t]{7}{*}{10} & 0.5 & 100 & 0.1 & 180.4745 & 51898.910 & 2.8789170 & 16.50961 \\
\hline & & & 0.5 & 2.504478 & 0.4792982 & -0.05866376 & 2.752959 \\
\hline & & & 1 & 1.566053 & 0.0519556 & -0.49187000 & 3.259029 \\
\hline & & & 2 & 1.247898 & 0.0088024 & -0.7519055 & 3.903483 \\
\hline & & & 3 & 1.158345 & 0.0034531 & -0.8483738 & 4.206997 \\
\hline & & & 4 & 1.116276 & 0.0018266 & -0.8989813 & 4.393305 \\
\hline & & & 5 & 1.091856 & 0.0011271 & -0.9301086 & 4.509344 \\
\hline
\end{tabular}


Table 2: $\mu_{1}{ }^{\prime}, \mathrm{V}(\mathrm{X}), \mathrm{S}(\mathrm{X}), \mathrm{K}(\mathrm{X})$ for the LL model.

\begin{tabular}{ccccc}
\hline $\mathrm{b}$ & $\mu_{1}{ }^{\prime}$ & $\mathrm{V}(\mathrm{X})$ & $\mathrm{S}(\mathrm{X})$ & $\mathrm{K}(\mathrm{X})$ \\
\hline 5 & 1.068959 & 0.1786323 & 2.48528 & 29.5562 \\
7.5 & 1.029853 & 0.0667170 & 1.33004 & 9.18867 \\
10 & 1.016641 & 0.0354009 & 0.93667 & 6.51021 \\
12.5 & 1.010606 & 0.0220617 & 0.72919 & 5.563852 \\
15 & 1.007348 & 0.01510236 & 0.598998 & 5.10838 \\
17.5 & 1.005391 & 0.01100040 & 0.509077 & 4.85121 \\
20 & 1.004124 & 0.00837532 & 0.443015 & 4.69083 \\
25 & 1.002637 & 0.00532522 & 0.352162 & 4.50848 \\
30 & 1.001830 & 0.00368497 & 0.292463 & 4.41214 \\
35 & 1.001344 & 0.00270154 & 0.250166 & 4.35495 \\
40 & 1.001029 & 0.00206550 & 0.218603 & 4.31819 \\
45 & 1.000813 & 0.00163045 & 0.194136 & 4.29314 \\
50 & 1.000658 & 0.00131982 & 0.172998 & 4.33383 \\
55 & 1.000544 & 0.00109017 & 0.158659 & 4.26215 \\
60 & 1.000457 & 0.00091569 & 0.14538480 & 4.25213 \\
65 & 1.000389 & 0.00078000 & 0.13416210 & 4.24442 \\
70 & 1.000336 & 0.00067239 & 0.12455090 & 4.23827 \\
75 & 1.000292 & 0.00058562 & 0.11622630 & 4.23332 \\
80 & 1.000257 & 0.000514623 & 0.10894580 & 4.22928 \\
85 & 1.000228 & 0.00045580 & 0.10252450 & 4.22593 \\
90 & 1.000203 & 0.00040652 & 0.09681852 & 4.22313 \\
95 & 1.000182 & 0.00036482 & 0.09171449 & 4.22077 \\
100 & 1.000164 & 0.00032925 & 0.08716923 & 3.74092 \\
\hline
\end{tabular}

\section{Copula}

\section{Bivariate WG-LL via Morgenstern family}

First, consider the $\mathrm{CDF}$ for Morgenstern model

$$
\left.F_{\lambda}\left(y_{1}, y_{2}\right)\right|_{(|\lambda| \leq 1)}=F_{1}\left(y_{1}\right) F_{2}\left(y_{2}\right)\left\{1+\lambda\left[1-F_{1}\left(y_{1}\right)\right]\left[1-F_{2}\left(y_{2}\right)\right]\right\} .
$$

Let

and

$$
F_{1}\left(y_{1}\right)=1-e^{-a_{1}\left[\left(1+y_{1}^{b_{1}}\right)^{\beta_{1}}-1\right]^{\alpha_{1}}}
$$

then we have new bivariate model as

$$
F_{2}\left(y_{2}\right)=1-e^{-a_{2}\left[\left(1+y_{2}^{b_{2}}\right)^{\beta_{2}}-1\right]^{\alpha_{2}}}
$$

$$
\begin{gathered}
\left.F_{\lambda}\left(y_{1}, y_{2}\right)\right|_{(|\lambda| \leq 1)}=\left(1-e^{-a_{1}\left[\left(1+y_{1}^{\ell_{1}}\right)^{\beta_{1}}-1\right]^{\alpha_{1}}}\right)\left(1-e^{-a_{2}\left[\left(1+y_{2}^{\ell_{2}}\right)^{\beta_{2}}-1\right]^{\alpha_{2}}}\right) \\
\times\left\{1+\lambda\left[e^{-a_{1}\left[\left(1+y_{1}^{\ell_{1}}\right)^{\beta_{1}}-1\right]^{\alpha_{1}}}\right]\left[e^{\left.-a_{2}\left[\left(1+y_{2}^{b_{2}}\right)^{\beta_{2}}-1\right]^{\alpha_{2}}\right]}\right] .\right.
\end{gathered}
$$

\section{Bivariate WG-LL via Clayton Copula}

Consider the following Clayton Copula

$$
C(u, v)=\left[u^{-\left(\delta_{1}+\delta_{2}\right)}+v^{-\left(\delta_{1}+\delta_{2}\right)}-1\right]^{-\frac{1}{\delta_{1}+\delta_{2}}} .
$$

Then, setting

$$
u=1-e^{-a_{1}\left[\left(1+x^{b_{1}}\right)^{\beta_{1}}-1\right]^{\alpha_{1}}}
$$


and

$$
v=1-e^{-a_{2}\left[\left(1+y^{b_{2}}\right)^{\beta_{2}}-1\right]^{\alpha_{2}}}
$$

The bivariate CDF can be written as

$$
C(u, v)=\left[\left(1-e^{-a_{1}\left[\left(1+x^{b_{1}}\right)^{\beta_{1}}-1\right]^{\alpha_{1}}}\right)^{-\left(\delta_{1}+\delta_{2}\right)}+\left(1-e^{-a_{2}\left[\left(1+y^{b_{2}}\right)^{\beta_{2}}-1\right]^{\alpha_{2}}}\right)^{-\left(\delta_{1}+\delta_{2}\right)}-1\right]^{-\frac{1}{\delta_{1}+\delta_{2}}} .
$$

The Multivariate extension via Copula of Clayton

The $d$-dimensional model can be expressed as

$$
H\left(x_{i}\right)=\left(\sum_{i=1}^{d}\left\{1-e^{-a_{i}\left[\left(1+x_{i}^{\ell_{i}}\right)^{\beta_{i}}-1\right]^{\alpha_{i}}}\right\}^{-\left(\delta_{1}+\delta_{2}\right)}+1-d\right)^{-\frac{1}{\delta_{1}+\delta_{2}}}
$$

where $x_{i}=x_{1}, x_{2}, \cdots, x_{d}$. For other copulas see Ali et al. (2020a), Ali et al. (2020b), Elgohari et al. (2021), Elgohari and Yousof (2020a,b and 2021).

\section{Simulations}

Assessing the behavior of the maximum likelihood estimations (MLEs) is discussed in this section. For this purpose, consider the following active algorithm:

1) Use

$$
y_{U}=\left(\left\{\left[-\frac{1}{a} \ln (1-U)\right]^{\frac{1}{\alpha}}+1\right\}^{\frac{1}{\beta}}-1\right)^{\frac{1}{b}}
$$

for generating 5000 group of size $n$ from the WG-LL model.

2) Get the MLEs for the 5000 groups.

3) Using the inverting the observed information matrix, compute the standard errors (SEs) of the MLEs for the 1000 samples, where the SEs).

4) Compute the biases $\left(B_{h}(n) \mid h=\alpha, \beta, a, b\right)$ and mean squared errors $\left(\operatorname{MSE}_{h}(n) \mid h=\alpha, \beta, a, b\right)$ given for $h=\alpha, \beta, a, b$. These steps must be repeated for $n=50,100, \ldots, 5000$ with $1=\alpha=b=\beta=a$ for getting the values of biases and the values of the $M S E_{h}$ for $a, b, \alpha$ and $n=50,100, \ldots, 5000$.

Figure 2, Figure 3, Figure 4 and Figure 5 (left charts) show how the four biases of $\alpha, \beta, a$ and $b$ vary with respect to $n \mid n=50,100, \ldots, 5000$. Figures $2,3,4$ and 5 (right charts) show how the four MSEs of $\alpha, \beta, a$ and $b$ vary with respect to $n \mid n=50,100, \ldots, 5000$. From Figure 2, 3 Figure, Figure 4 and Figure 5, the biases $f$ decrease to zero as $n \rightarrow \infty$, the valued of the obtained MSEs of $\alpha, \beta, a$ and $b$ decrease to zero as $n \rightarrow \infty$. Based on this assessment, the ML method performs well and can be used in estimating the model parameter. The following Section provide some useful real data applications using the ML method for comparing the competitive models. 

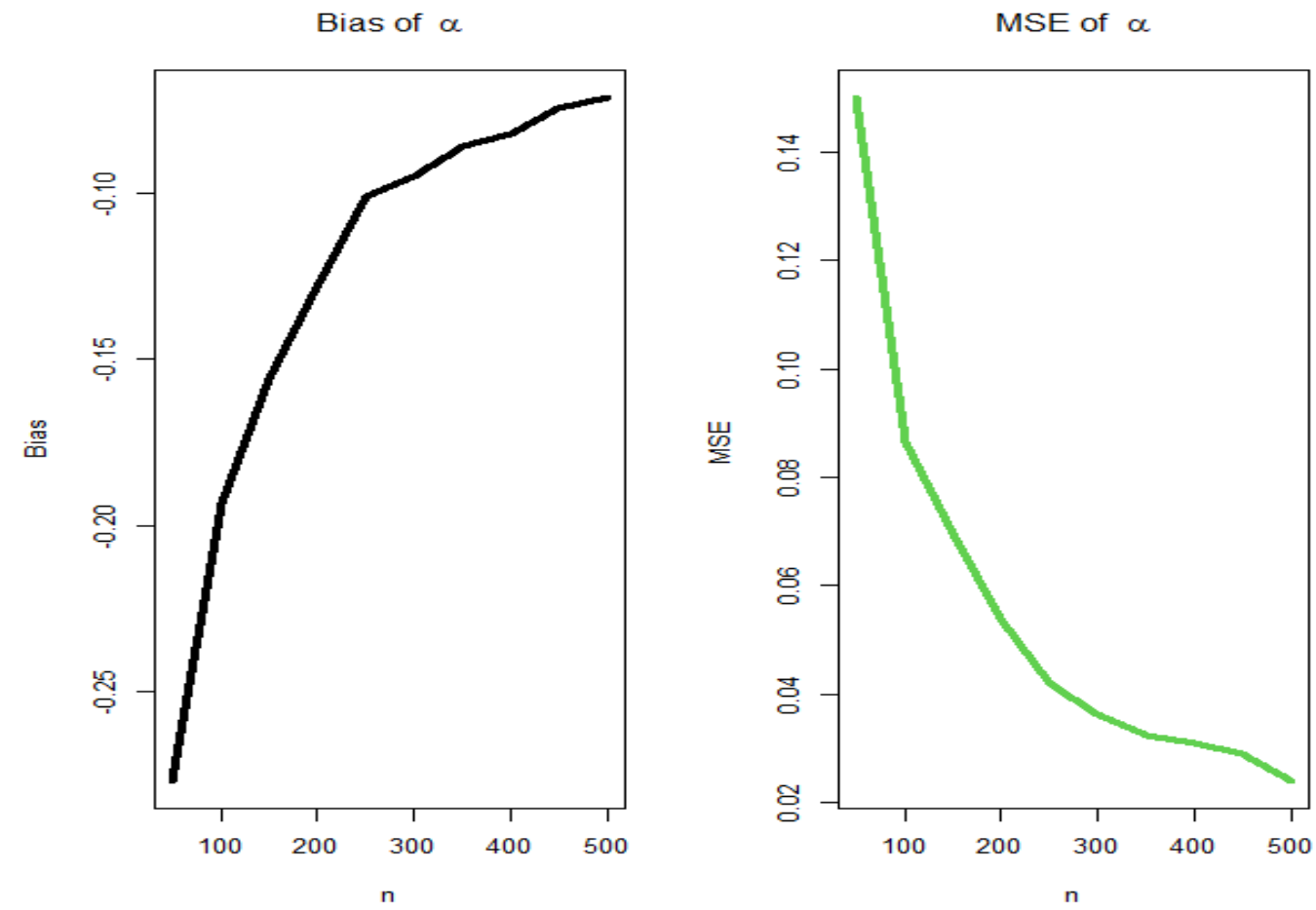

Figure 2: Bias and MSE for the parameter $\alpha$.
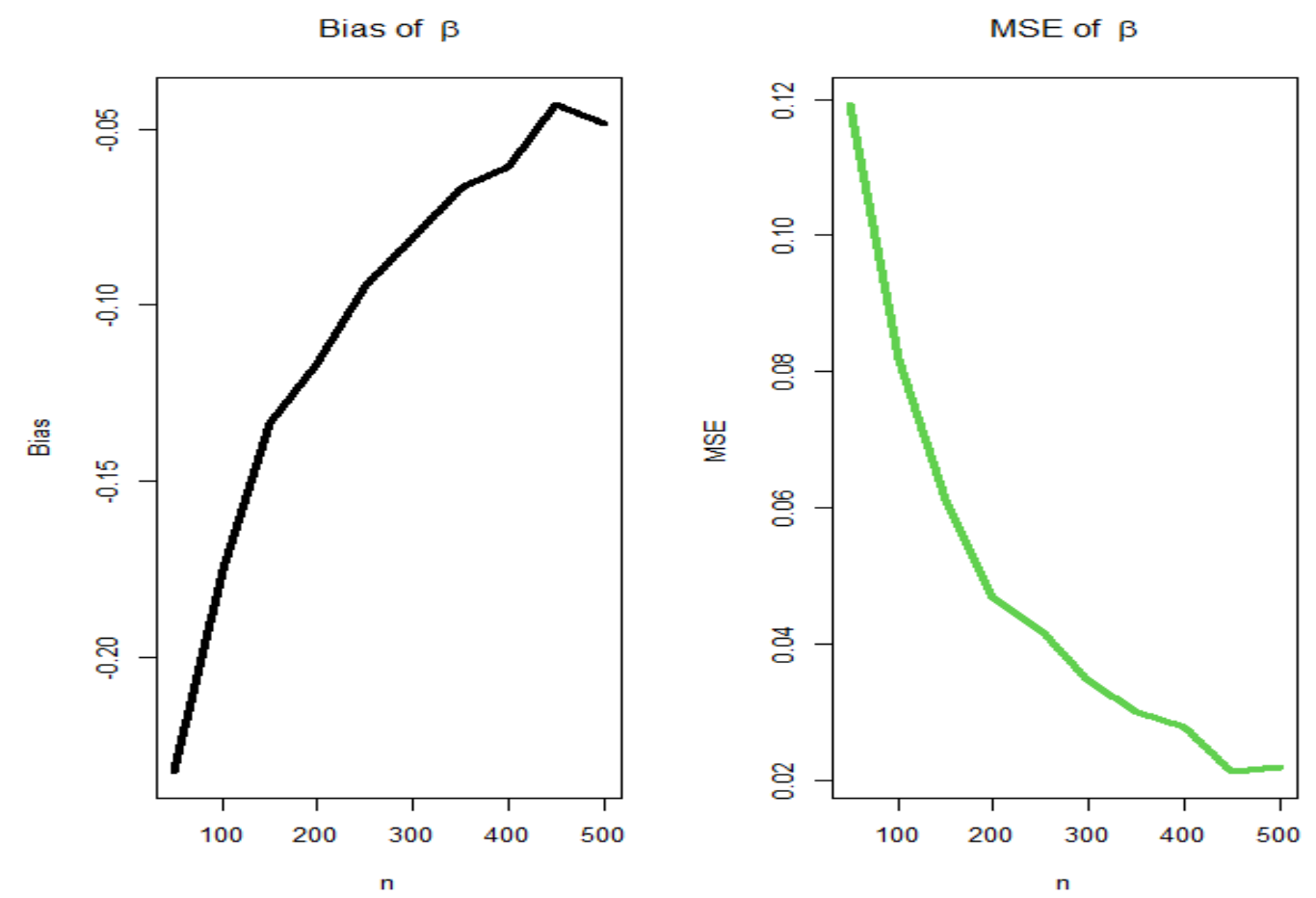

Figure 3: Bias and MSE for the parameter $\beta$. 

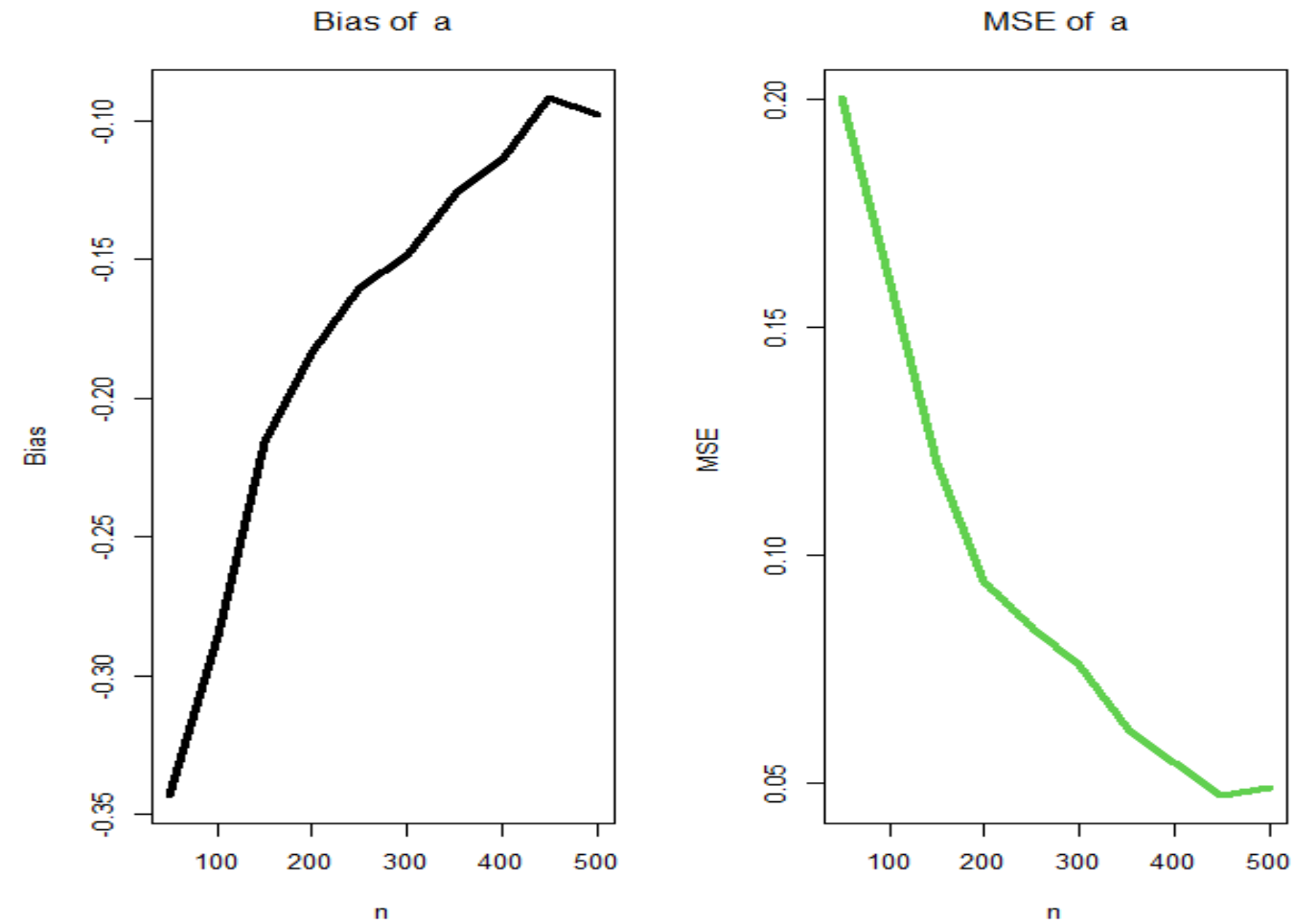

Figure 4: Bias and MSE for the parameter $a$.
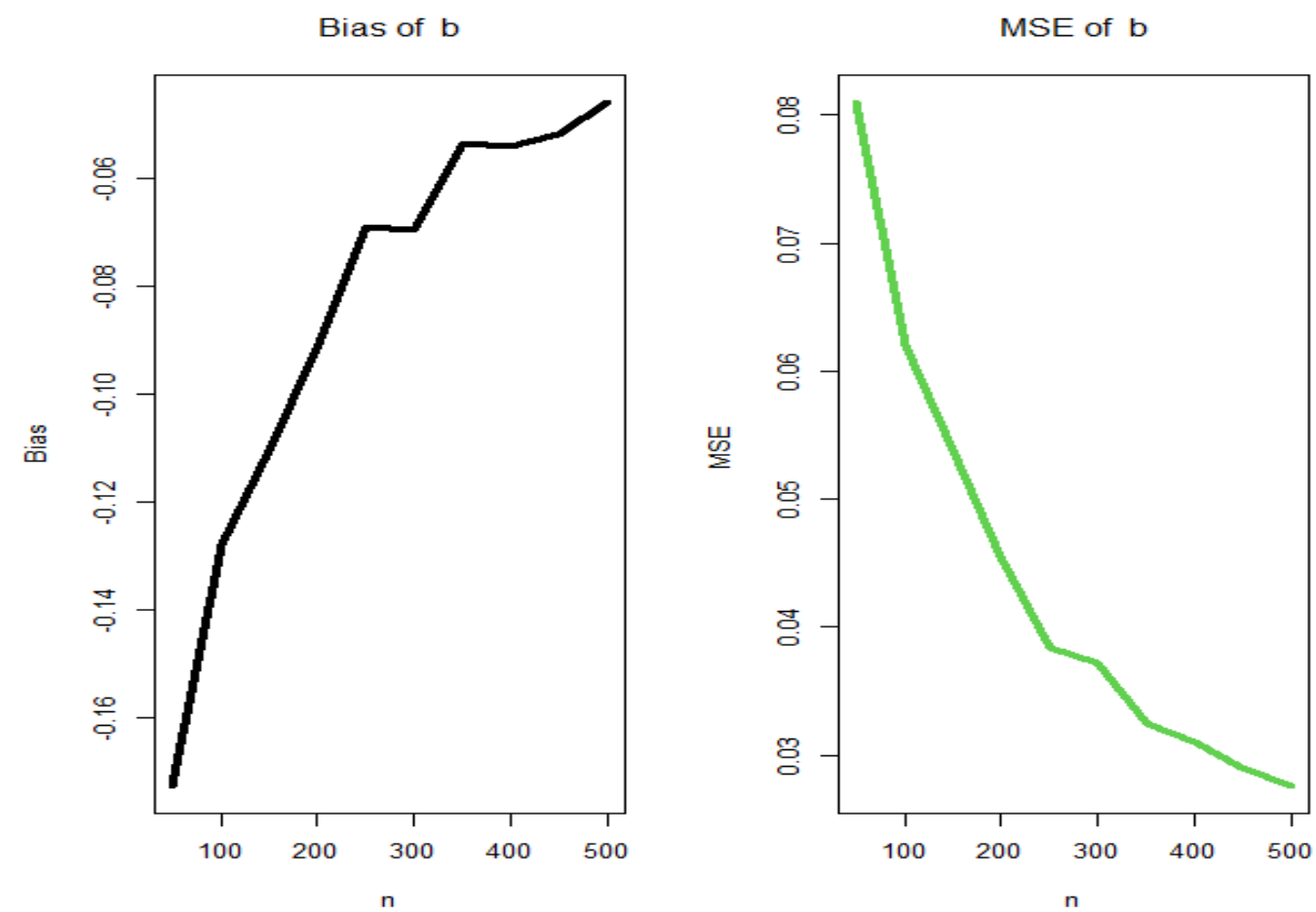

Figure 5: Bias and MSE for the parameter $b$. 


\section{Real data modeling and analysis}

In this part, some different real-life data sets are modeled and analyzed for demonstrating applied importance, applicable potentiality, and wide flexibility of the WG-LL model. For these data, we compare the WG-LL distribution, with other models such as the Burr model of the kind XII (BUXII), the WLL, Marchall-Olkan-BUXII (MOBUXII), Topp-Leone-BUXII (TLBUXII), Zografos-Balakrishnan-BUXII (ZOBBUXII), the beta-BUXII which has five parameters (FBBUXII), Beta BUXII b(BBUXII), Beta-exponentiated-BUXII (BEBUXII), five-parameters Kumaraswamy-BUXII (FKwBUXII), Kumaraswamy-BUXII (KwBUXII) and Weibull-log-logistic (WLL). All versions are recently modeled by Altun et al.(2018a) Yousof et al. (2018 and 2019) and Altun et al. (2018b). Other real-life datasets are in Aryal and Yousof (2017), Yousof et al. (2017), Hamedani et al. (2017,2018 and 2019), Merovci et al. (2017 and 2020), Korkmaz et al. (2018a), Korkmaz et al. (2018b), Nascimento et al. (2019), Alizadeh et al. (2020a,b), Korkmaz et al. (2020) and Karamikabir et al. (2020).

Real-life data set I: the breaking stress data. It consists of 100 observations of breaking stress of carbon fibers (in Gba) (see Nichols and Padgett (2006)). Real-life data set II: the survival times in days of 72 pigs from guinea which was infected with the virulent tubercle bacilli(Bjerkedal (1960)). Real-life data set III: the leukemia data. It represents the times of survival, in weeks, of 33 patients suffering from the wee-known acute myelogenous leukemia.

The total time test (TTT) plots (Aarset(1987)) for the three real data sets are presented in Figure 2. It is seen that the HRFs of data sets I, II are monotonical increasing and U-HRF for data set III. We consider the following goodnessof-fit statistics: the Akaike-criterion ( $\left.\mathrm{T}_{1}\right)$, Bayesian-criterion $\left(\mathrm{T}_{2}\right)$, consistent-criterion $\left(\mathrm{T}_{3}\right)$ and Hannan-Quinn criterion $\left(\mathrm{T}_{4}\right)$. Generally, the smaller these statistics are, the better the fit. Tables 3, 4 and 5 give the MLEs, standard errors (SEs), confidence interval (CIs95\%) with for the data set I, II and III. Tables 6, 7 and 8 give the statistics $\mathrm{T}_{1}$, $\mathrm{T}_{2}, \mathrm{~T}_{3}$, and $\mathrm{T}_{4}$ values for the data set I, II and III. Due to Table 6, Table 7 and Table 8 and Figure 3-6 the WG-LL model has the best results with small values of the $\mathrm{T}_{1}, \mathrm{~T}_{2}, \mathrm{~T}_{3}$, and $\mathrm{T}_{4}$.

Table 3: Estimation results for the data set $\mathbf{I}$.

\begin{tabular}{|c|c|c|}
\hline \multirow{2}{*}{$\frac{\text { Model }}{\operatorname{BUXII}(\beta, \alpha)}$} & \multicolumn{2}{|r|}{ Estimates, SEs and CI(95\%) } \\
\hline & Estimates & $5.941,0.187$ \\
\hline & SEs & $(1.2792),(0.0443)$ \\
\hline & $\mathrm{CI}(95 \%)$ & $(3.431,8.455),(0.100,0.277)$ \\
\hline \multirow{3}{*}{$\operatorname{MOBUXII}(\beta, \alpha, \gamma)$} & Estimates & $1.1921,4.8394,838.713$ \\
\hline & SEs & $(0.9522),(4.8961),(229.341)$ \\
\hline & $\mathrm{CI}(95 \%)$ & $(0,3.064),(0,14.438),(389.224,1288.246)$ \\
\hline \multirow[t]{3}{*}{$\operatorname{TLBUXII}(\beta, \alpha, \gamma)$} & Estimates & $1.3501,1.0614,13.7284$ \\
\hline & SEs & $(0.3788),(0.3844),(8.405)$ \\
\hline & $\mathrm{CI}(95 \%)$ & $(0.614,2.09),(0.313,1.811),(0,30.199)$ \\
\hline \multirow[t]{3}{*}{$\operatorname{KwBUXII}(\lambda, \theta, \alpha)}$, & Estimates & $48.1032,79.5162,0.3519,2.7305$ \\
\hline & SEs & $(19.340),(58.185),(0.098),(1.080)$ \\
\hline & $\mathrm{CI}(95 \%)$ & $(10.10,86.038),(0,193.568),(0.16,0.547),(0.62,4.845)$ \\
\hline \multirow[t]{3}{*}{$\operatorname{BBUXII}(\lambda, \theta, \alpha)}$, & Estimates & $359.7,260.1,0.1752,1.1233$ \\
\hline & SEs & $(57.9),(132,4),(0.013),(0.24)$ \\
\hline & $\mathrm{CI}(95 \%)$ & $(246,473),(0.96,519.24),(0.14,0.204),(0.65,1.66)$ \\
\hline \multirow{3}{*}{$\operatorname{BEBUXII}(\lambda, \theta, \alpha, \gamma)$} & Estimates & $0.3813,11.9492,0.9372,33.4026,1.7057$ \\
\hline & SEs & $(0.0783),(4.6353),(0.2674),(6.2871),(0.4789)$ \\
\hline & $\mathrm{CI}(95 \%)$ & $(0.2,0.535),(2.86,215),(0.41,1.55),(21,457),(0.8,2.67)$ \\
\hline \multirow[t]{3}{*}{$\operatorname{FBBUXII}(\lambda, \theta, \beta, \alpha, \gamma)$} & Estimates & $0.4212,0.834,6.2,1.67,3.452$ \\
\hline & SEs & $(0.01),(0.94),(2.31),(0.23),(1.96)$ \\
\hline & $\mathrm{CI}(95 \%)$ & $(0.4,0.44),(0.2 .7),(1.57,10.7),(1.23,2.1),(0,7)$ \\
\hline \multirow[t]{3}{*}{$\operatorname{FKwBUXII}(\lambda, \theta, \beta, \alpha, \gamma)$} & Estimates & $0.542,4.223,5.313,0.411,4.152$ \\
\hline & SEs & $(0.1375),(1.88),(2.32),(0.497),(1.9954)$ \\
\hline & $\mathrm{CI}(95 \%)$ & $(0.3,0.88),(0.53,7.96),(0.9,9.5),(0,1.74),(0.2,8.5)$ \\
\hline \multirow{3}{*}{$\operatorname{ZOBBUXII}(\lambda, \beta, \alpha)$} & Estimates & $123,0.368,139.23$ \\
\hline & SEs & $(243),(0.34),(319)$ \\
\hline & $\mathrm{CI}(95 \%)$ & $(0,599.401),(0,1.043),(0,763.599)$ \\
\hline \multirow[t]{2}{*}{$\operatorname{LL}(\mathrm{a})$} & Estimates & 1.6292359 \\
\hline & SEs & $(0.128801)$ \\
\hline
\end{tabular}




\begin{tabular}{|c|c|c|}
\hline \multirow{3}{*}{$\operatorname{ExpLL}(\beta, a)$} & $\mathrm{CI}(95 \%)$ & $(1.3611,1.847)$ \\
\hline & Estimates & $5.2214,2.31971$ \\
\hline & SEs & $(0.5981),(0.14966)$ \\
\hline \multirow{3}{*}{$\operatorname{WLL}(\beta, a)$} & $\mathrm{CI}(95 \%)$ & $(4.2,6.19),(2,2.59)$ \\
\hline & Estimates & $1.1159,0.709$ \\
\hline & SEs & $(6.511),(4.1259)$ \\
\hline \multirow{4}{*}{ WG-LL $(\beta, \alpha, b, a)$} & $\mathrm{CI}(95 \%)$ & $(0,16.09),(0,8.938)$ \\
\hline & Estimates & $2.206,0.667,0.137,1.581$ \\
\hline & SEs & $(2.509),(0.424),(0.351),(1.822)$ \\
\hline & $\mathrm{CI}(95 \%)$ & $(0,7.5),(0,1.5),(0,0.84),(0,5.1)$ \\
\hline \multicolumn{3}{|c|}{ Table 4: Estimation results for the data set II. } \\
\hline Model & & Estimates, SEs and CI(95\%) \\
\hline \multirow[t]{3}{*}{$\operatorname{BUXII}(\beta, \alpha)$} & Estimates & $3.1024,0.4649$ \\
\hline & SEs & $(0.5379),(0.0774)$ \\
\hline & $\mathrm{CI}(95 \%)$ & $(2.051,4.160),(0.310,0.624)$ \\
\hline \multirow{3}{*}{$\operatorname{MOBUXII}(\beta, \alpha, \gamma)$} & Estimates & $2.25911,1.5335,6.7603$ \\
\hline & SEs & $(0.8641),(0.9075),(4.5871)$ \\
\hline & $\mathrm{CI}(95 \%)$ & $(0.571,3.955),(0,3.311),(0,15.757)$ \\
\hline \multirow[t]{3}{*}{$\operatorname{TLBUXII}(\beta, \alpha, \gamma)$} & Estimates & $2.396,0.4584,1.7964$ \\
\hline & SEs & $(0.91),(0.24),(0.92)$ \\
\hline & $\mathrm{CI}(95 \%)$ & $(0.6,4.18),(0,0.95),(0.002,3.6)$ \\
\hline \multirow[t]{3}{*}{$\operatorname{KwBUXII}(\lambda, \theta, \beta, \alpha)$} & Estimates & $14.1049,7.42,0.53,2.273$ \\
\hline & SEs & $(10.81),(11.85),(0.28),(0.991)$ \\
\hline & $\mathrm{CI}(95 \%)$ & $(0,35.3),(0.30 .7),(0,1.1),(0.33,4.26)$ \\
\hline \multirow[t]{3}{*}{$\operatorname{BBUXII}(\lambda, \theta, \beta, \alpha)$} & Estimates & $2.6,6.0580,1.8,0.29434$ \\
\hline & SEs & (1.86), (10.39), (0.96), (0.47) \\
\hline & $\mathrm{CI}(95 \%)$ & $(0,6.3),(0,26.5),(0,3.7),(0,1.2)$ \\
\hline \multirow{3}{*}{$\operatorname{BEBUXII}(\lambda, \theta, \beta, \alpha, \gamma)$} & Estimates & $1.88,2.99,1.781,1.342,0.573$ \\
\hline & SEs & $(0.09),(1.73),(0.7),(0.82),(0.33)$ \\
\hline & $\mathrm{CI}(95 \%)$ & $(1.7,2.1),(0,6.4),(0.40,3.19),(0,3),(0,1.22)$ \\
\hline \multirow[t]{3}{*}{$\operatorname{FBBUXII}(\lambda, \theta, \beta, \alpha, \gamma)$} & Estimates & $0.62,0.55,3.838,1.38,1.67$ \\
\hline & SEs & $(0.54),(1.01),(2.79),(2.31),(0.44)$ \\
\hline & $\mathrm{CI}(95 \%)$ & $(0,1.73),(0,2.53),(0,9.31),(0,5.99),(0.8,4.55)$ \\
\hline \multirow[t]{3}{*}{$\operatorname{FKwBUXII}(\lambda, \theta, \beta, \alpha, \gamma)$} & Estimates & $0.5583,0.308,3.9991,2.1312,1.4754$ \\
\hline & SEs & $(0.44),(0.3143),(2.08),(1.83),(0.364)$ \\
\hline & $\mathrm{CI}(95 \%)$ & $(0,1.29),(0,0.89),(0,3.12),(0,5.69),(0.76,2.3)$ \\
\hline \multirow[t]{3}{*}{ LL(a) } & Estimates & 2.27532 \\
\hline & SEs & $(0.22327)$ \\
\hline & $\mathrm{CI}(95 \%)$ & $(1.92,2.78)$ \\
\hline \multirow[t]{3}{*}{$\operatorname{ExpLL}(\beta, a)$} & Estimates & $1.9513,2.254$ \\
\hline & SEs & $(0.2288),(0.20679)$ \\
\hline & $\mathrm{CI}(95 \%)$ & $(1.51,2.33),(2.14,2.90)$ \\
\hline \multirow[t]{3}{*}{$\operatorname{WLL}(\beta, a)$} & Estimates & $0.78545,1.25401$ \\
\hline & SEs & $(0.00),(0.00)$ \\
\hline & $\mathrm{CI}(95 \%)$ &,---- \\
\hline \multirow[t]{3}{*}{ WG-LL $(\beta, \alpha, b, a)$} & Estimates & $0.869,0.328,0.879,3.486$ \\
\hline & SEs & $(0.729),(0.262),(1.477),(2.886)$ \\
\hline & $\mathrm{CI}(95 \%)$ & $(0,2.33),(0,0.852),(0,3.879),(0,9.1)$ \\
\hline
\end{tabular}

Table 5: Estimation results for the data set III.

\begin{tabular}{ccc}
\hline Model & & Estimates, SEs and CI (95\%) \\
\hline BUXII $(\beta, \alpha)$ & Estimates & $58.7110,0.0062$ \\
& SEs & $(42.3821),(0.0046)$ \\
MOBUXII $(\beta, \alpha, \gamma)$ & Es $(95 \%)$ & $(0,141.782),(0,0.014)$ \\
& Estimates & $11.8381,0.0783,12.2510$
\end{tabular}




\begin{tabular}{|c|c|c|}
\hline \multirow{5}{*}{$\operatorname{TLBUXII}(\beta, \alpha, \gamma)$} & SEs & $(4.3681),(0.0129),(7.771)$ \\
\hline & $\mathrm{CI}(95 \%)$ & $(0,141.8),(0,0.011),(0,27.50)$ \\
\hline & Estimates & $0.2814,1.8823,50.2147$ \\
\hline & SEs & $(0.29),(2.40),(176.5)$ \\
\hline & $\mathrm{CI}(95 \%)$ & $(0,0.855),(0,6.599),(0,396)$ \\
\hline \multirow{3}{*}{$\operatorname{KwBUXII}(\lambda, \theta, \beta, \alpha)$} & Estimates & $9.201,36.428,0.242,0.941$ \\
\hline & SEs & (10.06), (35.651), (0.168), (1.0455) \\
\hline & $\mathrm{CI}(95 \%)$ & $(0,28.9123),(0,106.3),(0,0.57),(0,3)$ \\
\hline \multirow[t]{3}{*}{$\operatorname{BBUXII}(\lambda, \theta, \beta, \alpha)$} & Estimates & $96.1,52.12,0.104,1.2278$ \\
\hline & SEs & (41.2), (33.49), (0.02), (0.33) \\
\hline & $\mathrm{CI}(95 \%)$ & $(15.4,177),(0,118),(0.6,0.15),(0.59,2)$ \\
\hline \multirow{3}{*}{$\operatorname{BEBUXII}(\lambda, \theta, \beta, \alpha, \gamma)$} & Estimates & $0.087,5.01,1.56,31.27,0.323$ \\
\hline & SEs & (0.09), (3.85), (0.012), (12.94), (0.0344) \\
\hline & $\mathrm{CI}(95 \%)$ & $(0,0.33),(0,12.66),(1.5,1.66),(5.9,56.58),(0.3,0.44)$ \\
\hline \multirow{3}{*}{$\operatorname{FBBUXII}(\lambda, \theta, \beta, \alpha, \gamma)$} & Estimates & $15.1943,32.0482,0.233,0.5801,21.8555$ \\
\hline & SEs & (11.59), (9.868), (0.092), (0.07), (35.55) \\
\hline & $\mathrm{CI}(95 \%)$ & $(0,37.77),(12.7,51),(0.05,0.4),(0.45,0.7),(0,91.5)$ \\
\hline \multirow{3}{*}{$\operatorname{FKwBUXII}(\lambda, \theta, \beta, \alpha, \gamma)$} & Estimates & $14.73,15.28,0.29,0.84,0.0342$ \\
\hline & SEs & (12.39), (18.87), (0.22), (0.85), (0.08) \\
\hline & $\mathrm{CI}(95 \%)$ & $(0,39),(0,52.3),(0,0.7),(0,2.5),(0,0.2)$ \\
\hline \multirow[t]{3}{*}{$\operatorname{ZOBBUXII}(\lambda, \beta, \alpha, \gamma)$} & Estimates & $41.9733,0.1572,44.2632$ \\
\hline & SEs & $(38.7875),(0.0824),(47.65)$ \\
\hline & $\mathrm{CI}(95 \%)$ & $(0,118),(0,0.32),(0,137.65)$ \\
\hline \multirow[t]{3}{*}{ LL(a) } & Estimates & 0.507323 \\
\hline & SEs & $(0.07090)$ \\
\hline & $\mathrm{CI}(95 \%)$ & $(0.366,0.644)$ \\
\hline \multirow[t]{3}{*}{$\operatorname{ExpLL}(\beta, a)$} & Estimates & $5.5941,0.76478$ \\
\hline & SEs & $(1.18343),(0.0933)$ \\
\hline & $\mathrm{CI}(95 \%)$ & $(3.1,7.99),(0.5,0.888)$ \\
\hline \multirow[t]{3}{*}{$\operatorname{WLL}(\beta, a)$} & Estimates & $1.0699,0.2245$ \\
\hline & SEs & $(0.000),(0.000)$ \\
\hline & $\mathrm{CI}(95 \%)$ &,---- \\
\hline \multirow{3}{*}{ WG-LL $(\beta, \alpha, b, a)$} & Estimates & $0.643,0.160,0.091,6.701$ \\
\hline & SEs & $(0.253),(0.076),(0.048),(0.009)$ \\
\hline & $\mathrm{CI}(95 \%)$ & $(0.1,1.1),(0.02,0.3),(0,0.186),(6.68,6.718)$ \\
\hline
\end{tabular}

Table 4: $\mathrm{T}_{1}, \mathrm{~T}_{2}, \mathrm{~T}_{3}$, and $\mathrm{T}_{4}$ values for the data set $\mathbf{I}$.

\begin{tabular}{|c|c|}
\hline Model & $\mathrm{T}_{1}, \mathrm{~T}_{2}, \mathrm{~T}_{3}$, and $\mathrm{T}_{4}$ \\
\hline BUXII & $382.942,388.155,383.063,385.053$ \\
\hline WLL & $510.693,515.911,510.820,512.844$ \\
\hline TLBUXII & $323.524,331.354,323.771,326.701$ \\
\hline MOBUXII & $305.781,313.614,306.030,308.960$ \\
\hline FBBUXII & $304.260,317.311,304.892,309.564$ \\
\hline ExpLL & $325.931,331.144,326.066,328.040$ \\
\hline BBUXII & $305.644,316.060,306.063,309.853$ \\
\hline KwBUXII & $303.761,314.201,304.185,308.001$ \\
\hline BEBUXII & $305.822,318.845,306.462,311.093$ \\
\hline LL & $469.633,472.233,469.670,470.680$ \\
\hline FKwBUXII & $305.503,318.551,306.145,310.802$ \\
\hline ZOBBUXII & $302.960,310.781,303.214,306.130$ \\
\hline WG-LL & $\mathbf{2 9 0 . 7 5 0 , 3 0 1 . 1 7 3 , 2 9 1 . 1 7 4 , 2 9 4 . 9 7 0}$ \\
\hline
\end{tabular}


Table 6: $\mathrm{T}_{1}, \mathrm{~T}_{2}, \mathrm{~T}_{3}$, and $\mathrm{T}_{4}$ values for the data set II

\begin{tabular}{|c|c|}
\hline Model & $\mathrm{T}_{1}, \mathrm{~T}_{2}, \mathrm{~T}_{3}$, and $\mathrm{T}_{4}$ \\
\hline BUXII & $209.600,214.154,209.771,211.401$ \\
\hline LL & $231.850,234.134,231.914,232.765$ \\
\hline FBBUXII & $206.803,218.204,207.716,211.303$ \\
\hline ExpLL & $207.833,212.385,208.003,209.644$ \\
\hline MOBUXII & $209.743,216.564,210.091,212.443$ \\
\hline TLBUXII & $211.803,218.633,212.154,214.520$ \\
\hline WLL & $270.441,274.981,270.602,272.230$ \\
\hline KwBUXII & $208.760,217.865,209.366,212.380$ \\
\hline BBUXII & $210.443,219.545,211.036,214.060$ \\
\hline FKwBUXII & $206.501,217.905,207.413,211.000$ \\
\hline BEBUXII & $212.103,223.503,213.001,216.600$ \\
\hline WG-LL & $\mathbf{2 0 5 . 8 6 2 , 2 1 4 . 9 7 0 , 2 0 6 . 4 6 0 , 2 0 9 . 4 9 0}$ \\
\hline
\end{tabular}

Table 8: $\mathrm{T}_{1}, \mathrm{~T}_{2}, \mathrm{~T}_{3}$, and $\mathrm{T}_{4}$ values for the data set III.

\begin{tabular}{|c|c|}
\hline Model & $\mathrm{T}_{1}, \mathrm{~T}_{2}, \mathrm{~T}_{3}$, and $\mathrm{T}_{4}$ \\
\hline BUXII & $328.203,331.193,328.601,329.194$ \\
\hline LL & $362.711,364.200,362.833,363.215$ \\
\hline MOBUXII & $315.541,320.010,316.370,317.043$ \\
\hline TLBUXII & $316.264,320.733,317.092,317.761$ \\
\hline BBUXII & $316.461,322.454,317.893,318.471$ \\
\hline KwBUXII & $317.363,323.301,318.793,319.343$ \\
\hline ExpLL & $315.4652,318.450,315.850,316.47$ \\
\hline FBBUXII & $317.863,325.342,320.081,320.364$ \\
\hline BEBUXII & $317.580,325.062,319.801,320.090$ \\
\hline FKwBUXII & $317.760,325.213,319.982,320.260$ \\
\hline ZOBBUXII & $313.862,318.354,314.39,315.3603$ \\
\hline WLL & $378.8212,381.7902,379.27,379.84$ \\
\hline WG-LL & $\mathbf{3 1 0 . 9 0 0 , 3 1 6 . 8 9 1 , 3 1 2 . 3 3 2 , 3 1 2 . 9 2 3}$ \\
\hline
\end{tabular}

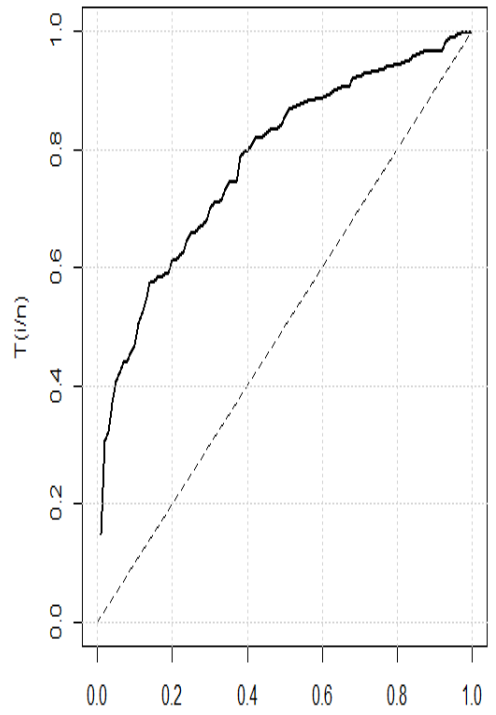

in

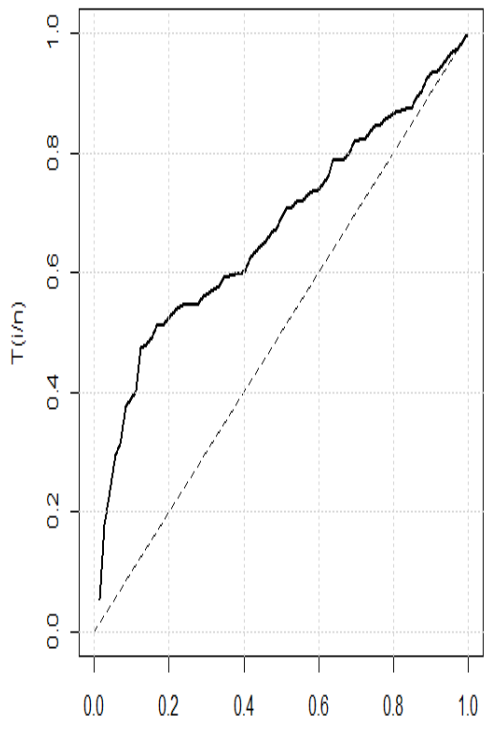

i/n

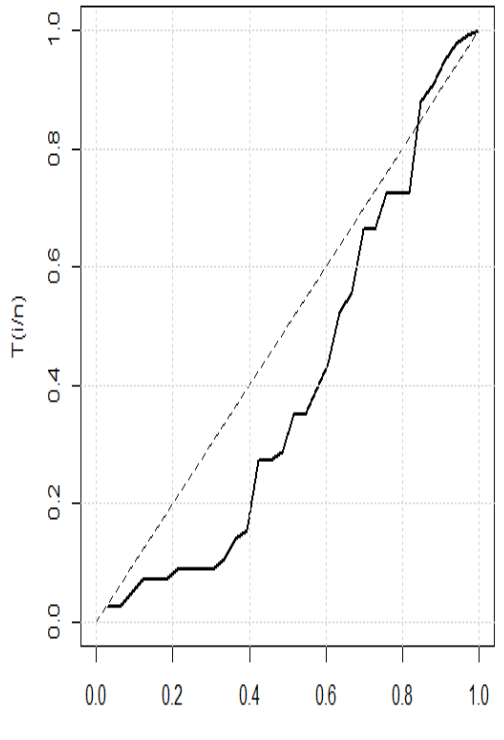

i/n 
Figure 6: TTT plots.

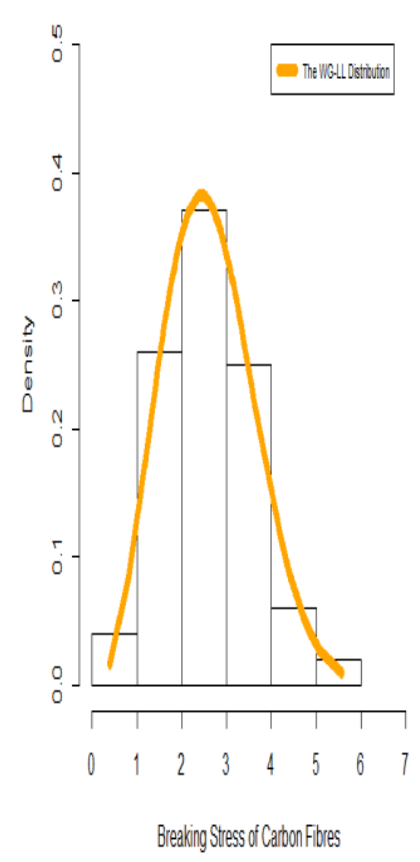

Estimated CDF (Survival Times Data)

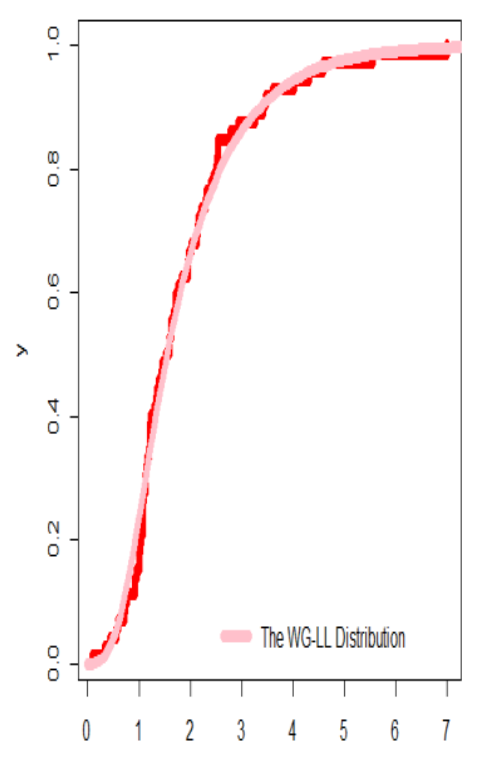

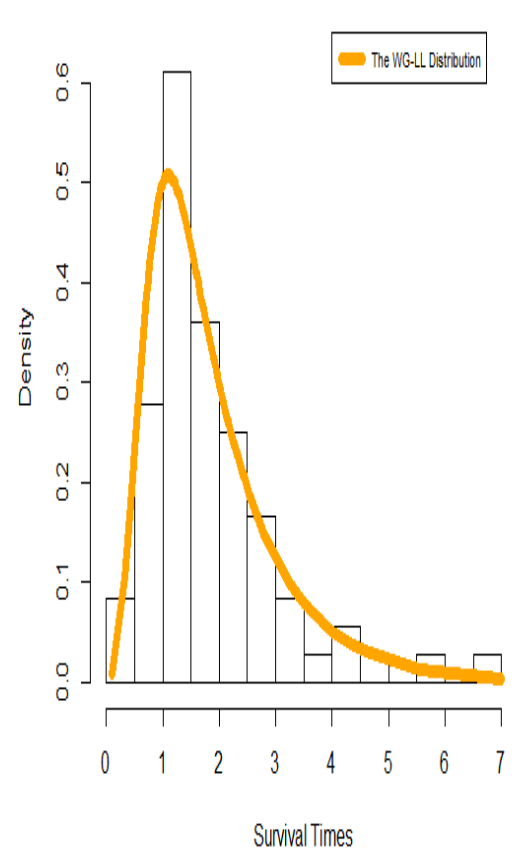

Figure 7: Estimated PDFs.

Estimated CDF (Leukaemia Data)

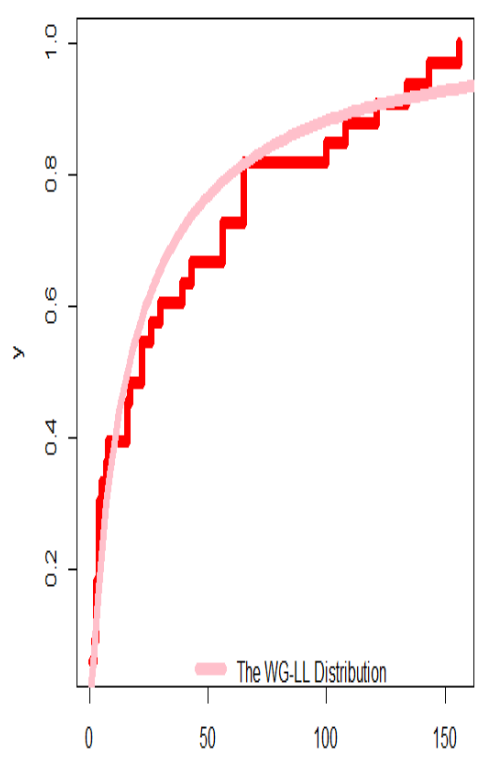

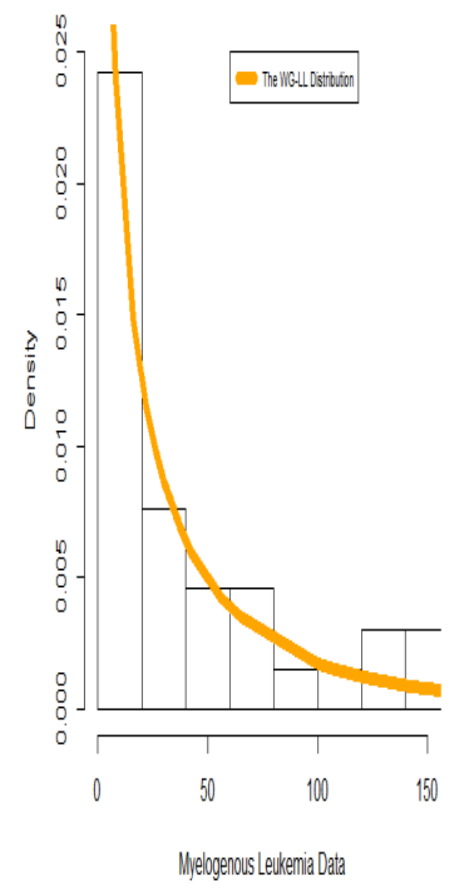

Estimated CDF (Breaking Stress Data)

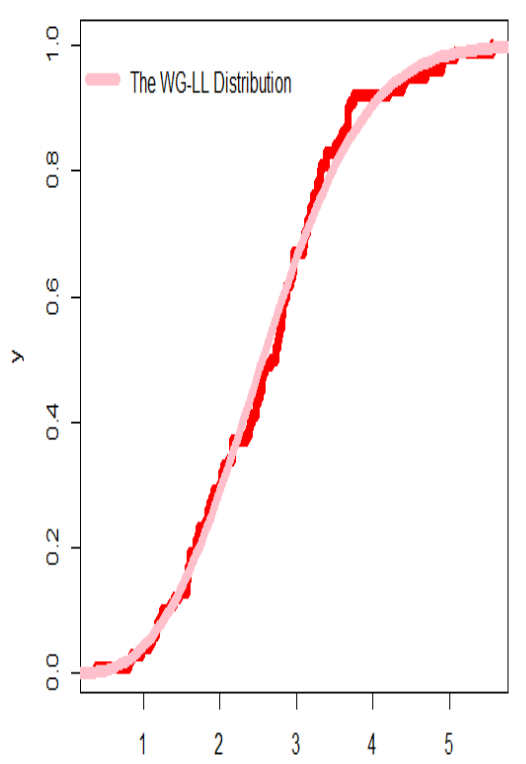

Figure 8: Estimated CDFs. 


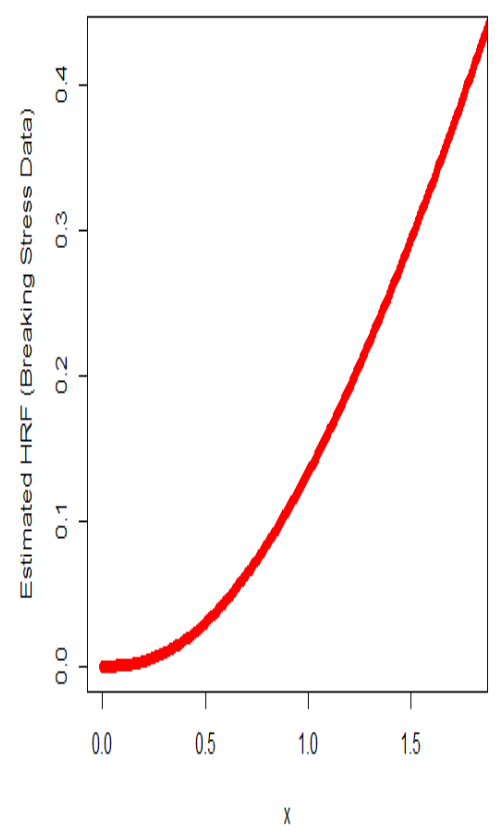

Kaplan-Meier Survival Plot for Data Setl

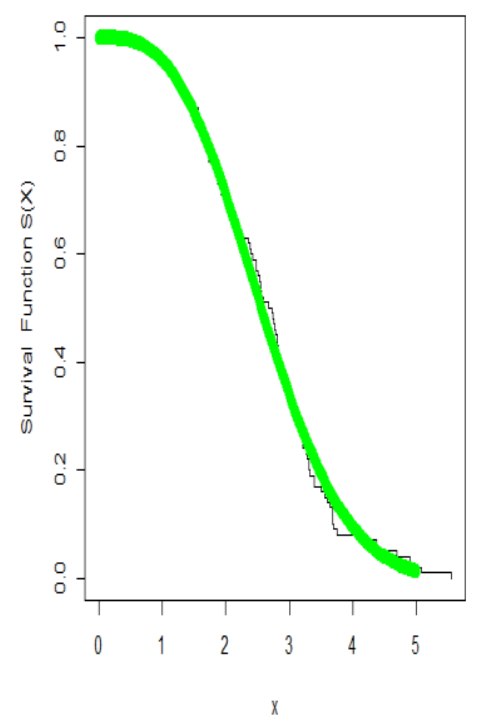

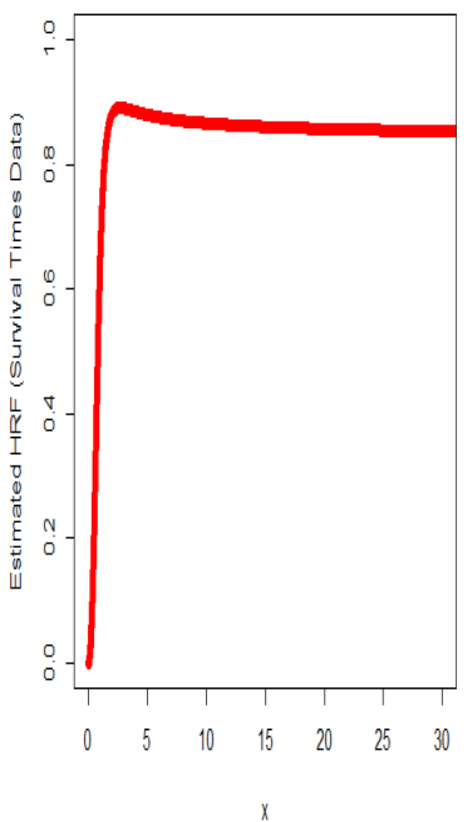

Figure 9: Estimated HRFs.

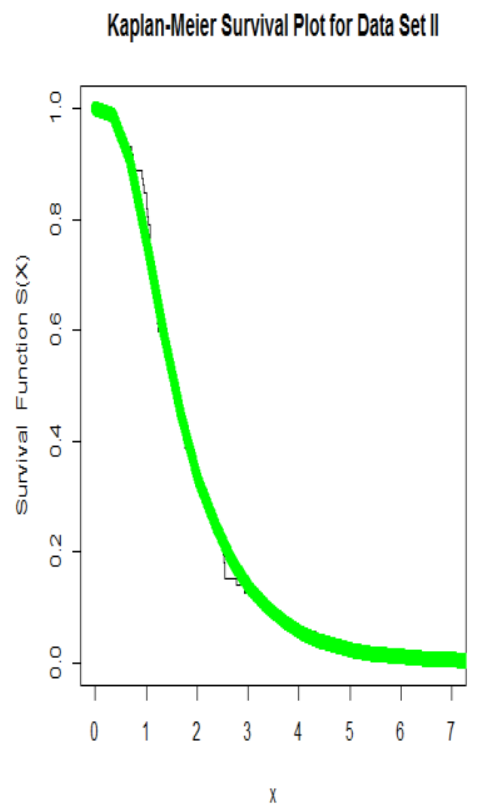

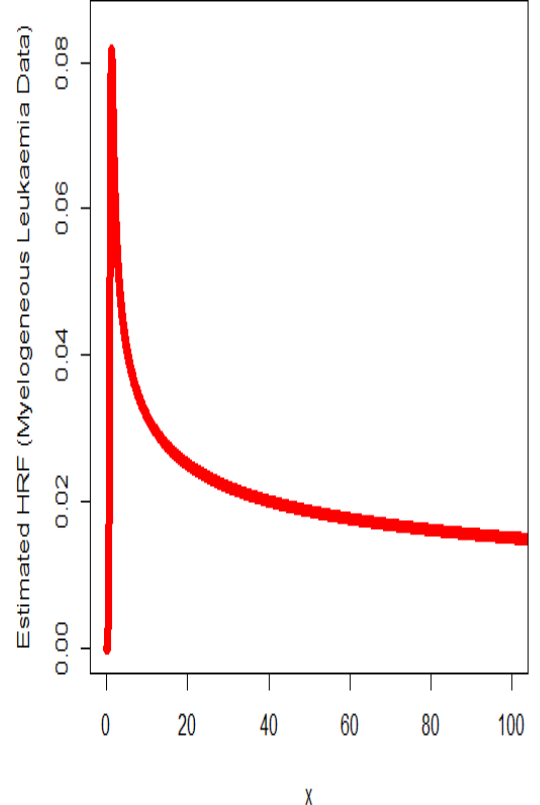

Kaplan-Meier Survival Plot for Data Set III

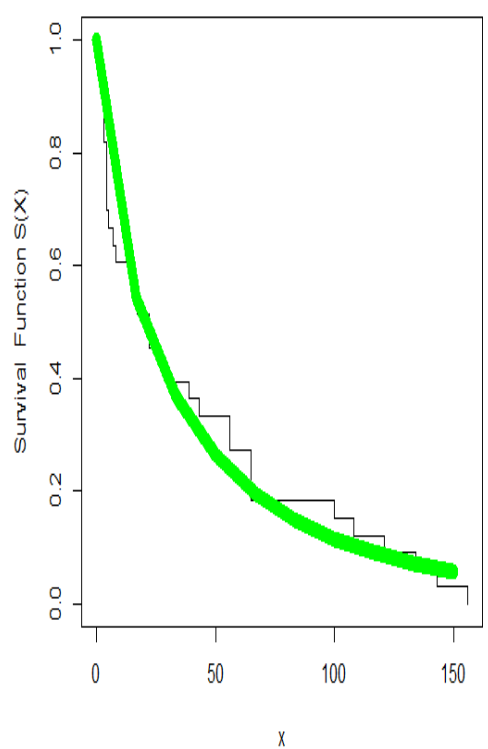

Figure 10: Kaplan-Meier survival plots.

\section{Concluding remarks}

In the present paper, we introduced a novel continuous log-logistic model. Several of its main characteristic properties such as the moments, the generating function, the weighted moments, the reversed residual life are mathematically derived. Numerical analysis for the skewness and the kurtosis is presented and useful comments are added. For the new log-logistic model, the skewness $\in(-1.081,16.74)$. However, for the standard log-logistic model, the skewness 
$\in(0.087,2.4853)$. hence, the novel model can be negative skewed and positive skewed while the standard model can only be negative skewness. For the new log-logistic model, kurtosis $\in(3.245089,702.498)$. However, for the standard $\log$-logistic model, kurtosis $\in(3.741,29.56)$. The new PDF can be unimodal, symmetric, or left skewed. The new failure rate can be "bathtub or $\mathbf{U}$-failure rate", "increasing failure rate", "decreasing-constant failure rate", "J-failure rate", "constant failure rate " and "decreasing failure rate".

Many bivariate and extensions are derived. To assess the estimators, we performed a graphical simulation. Three different real-life data are modeled under some statistical tests. For all these real-life datasets, we compare the novel function with many relevant extensions. The new model is better than all other competitive models in modeling breaking stress data, survival times data and leukemia data.

Future points:

1- Presenting a novel discrete model for modeling count real-life data (see Aboraya et al. (2020), Chesneau et al. (2021), Ibrahim et al. (2021) and Yousof et al. (2021) for more details).

2- Appling the Nikulin-Rao-Robson and Bagdonavičius-Nikulin tests (see Ibrahim et al. (2019), Goual et al. (2019, 2020), Mansour et al. (2020a,b,c,d,e,f), Yadav et al. (2020), Goual and Yousof (2020) and Aidi et al. (2021), among others.).

\section{References}

1. Aarset, M. V. (1987). How to identify a bathtub hazard rate. IEEE Transactions on Reliability, 36(1), 106-108.

2. Aboraya, M., M. Yousof, H. M., Hamedani, G. G. and Ibrahim, M. (2020). A new family of discrete distributions with mathematical properties, characterizations, Bayesian and non-Bayesian estimation methods. Mathematics, $8,1648$.

3. Aidi, K., Butt, N. S., Ali, M. M., Ibrahim, M., Yousof, H. M. and Shehata, W. A. M. (2021). A Modified Chisquare Type Test Statistic for the Double Burr X Model with Applications to Right Censored Medical and Reliability Data. Pakistan Journal of Statistics and Operation Research, 17(3), 615-623.

4. Ali, M. M., Ibrahim, M. and Yousof, H. M. (2021a). Expanding the Burr X model: properties, copula, real data modeling and different methods of estimation. Optimal Decision Making in Operations Research \& Statistics: Methodologies and Applications, CRC Press, 26-49.

5. Ali, M. M., Yousof, H. M. and Ibrahim, M. (2021b). A new version of the generalized Rayleigh distribution with copula, properties, applications and different methods of estimation. Optimal Decision Making in Operations Research \& Statistics: Methodologies and Applications, CRC Press, 1-25.

6. Alizadeh, M., Jamal, F., Yousof, H. M., Khanahmadi, M. and Hamedani, G. G. (2020a). Flexible Weibull generated family of distributions: characterizations, mathematical properties and applications. University Politehnica of Bucharest Scientific Bulletin-Series A-Applied Mathematics and Physics, 82(1), 145-150.

7. Alizadeh, M., Yousof, H. M., Jahanshahi, S. M. A., Najibi, S. M. and Hamedani, G. G. (2020b). The transmuted odd log-logistic-G family of distributions. Journal of Statistics and Management Systems, 23(4), 1-27.

8. Altun, E., Yousof, H. M. and Hamedani, G. G. (2018 a). A new log-location regression model with influence diagnostics and residual analysis. Facta Universitatis, Series: Mathematics and Informatics, 33(3), 417-449.

9. Altun, E., Yousof, H. M., Chakraborty, S. and Handique, L. (2018 b). Zografos-Balakrishnan Burr XII distribution: regression modeling and applications. International Journal of Mathematics and Statistics, 19(3), 4670.

10. Aryal, G. R. and Yousof, H. M. (2017). The exponentiated generalized-G Poisson family of distributions. Economic Quality Control, 32(1), 1-17.

11. Bjerkedal, T. (1960). Acquisition of resistance in Guinea pigs infected with different doses of virulent tubercle bacilli. American Journal of Hygiene, 72, 130--148.

12. Burr, I. W. (1942). Cumulative frequency functions. Annals of Mathematical Statistics, 13, 215-232.

13. Burr, I. W. (1968). On a general system of distributions, III.The simplerange.Journal of the American Statistical Association, 63, 636-643.

14. Burr, I. W. (1973). Parameters fora general system of distributions to match a grid of 3 and 4.Communications in Statistics, 2, 1-21.

15. Burr, I. W. and Cislak, P. J. (1968). On a general system of distributions: I. Its curve-shaped characteristics; II. The sample median. Journalof the American Statistical Association, 63, 627-635.

16. Chesneau, C., Yousof, H. M., Hamedani, G. and Ibrahim, M. (2022). A New One-parameter Discrete Distribution: The Discrete Inverse BurrDistribution: Characterizations, Properties, Applications, Bayesian and Non-Bayesian 
Estimations. Statistics, Optimization \& Information Computing, forthcoming.

17. Elgohari, H. and Yousof, H. M. (2020a). A Generalization of Lomax Distribution with Properties, Copula and Real Data Applications. Pakistan Journal of Statistics and Operation Research, 16(4), 697-711.

18. Elgohari, H. and Yousof, H. M. (2021). A New Extreme Value Model with Different Copula, Statistical Properties and Applications. Pakistan Journal of Statistics and Operation Research, 17(4), 1015-1035.

19. Elgohari, H. and Yousof, H. M. (2020b). New Extension of Weibull Distribution: Copula, Mathematical Properties and Data Modeling. Statistics, Optimization \& Information Computing, 8(4), 972-993.

20. Elgohari, H., Ibrahim, M. and Yousof, H. M. (2021). A New Probability Distribution for Modeling Failure and Service Times: Properties, Copulas and Various Estimation Methods. Statistics, Optimization \& Information Computing, 8(3), 555-586.

21. Gradshteyn, I. S. and Ryzhik, I. M. (2000). Table of Integrals, Series and Products (sixth edition).San Diego: Academic Press.

22. Goual, H., Yousof, H. M. and Ali, M. M. (2019). Validation of the odd Lindley exponentiated exponential by a modified goodness of fit test with applications to censored and complete data. Pakistan Journal of Statistics and Operation Research, 15(3), 745-771.

23. Goual, H. and Yousof, H. M. (2020). Validation of Burr XII inverse Rayleigh model via a modified chi-squared goodness-of-fit test. Journal of Applied Statistics, 47(3), 393-423.

24. Goual, H., Yousof, H. M. and Ali, M. M. (2020). Lomax inverse Weibull model: properties, applications, and a modified Chi-squared goodness-of-fit test for validation. Journal of Nonlinear Sciences \& Applications, 13(6), 330-353.

25. Hamedani, G. G., Altun, E, Korkmaz, M. C., Yousof, H. M. and Butt, N. S. (2018). A new extended G family of continuous distributions with mathematical properties, characterizations and regression modeling. Pak. J. Stat. Oper. Res., 14(3), 737-758.

26. Hamedani, G. G. Rasekhi, M., Najib, S. M., Yousof, H. M. and Alizadeh, M., (2019). Type II general exponential class of distributions. Pak. J. Stat. Oper. Res., XV (2), 503-523.

27. Hamedani, G. G. Yousof, H. M., Rasekhi, M., Alizadeh, M., Najibi, S. M. (2017). Type I general exponential class of distributions. Pak. J. Stat. Oper. Res., XIV (1), 39-55.

28. Ibrahim, M., Ali, M. M. and Yousof, H. M. (2021). The discrete analogue of the Weibull G family: properties, different applications, Bayesian and non-Bayesian estimation methods. Annals of Data Science, forthcoming.

29. Ibrahim, M., Yadav, A. S., Yousof, H. M., Goual, H. and Hamedani, G. G. (2019). A new extension of Lindley distribution: modified validation test, characterizations and different methods of estimation. Communications for Statistical Applications and Methods, 26(5), 473-495.

30. Karamikabir, H., Afshari, M., Yousof, H. M., Alizadeh, M. and Hamedani, G. (2020). The Weibull Topp-Leone Generated Family of Distributions: Statistical Properties and Applications. Journal of The Iranian Statistical Society, 19(1), 121-161.

31. Korkmaz, M. Ç., Altun, E., Yousof, H. M. and Hamedani, G. G. (2020). The Hjorth's IDB Generator of Distributions: Properties, Characterizations, Regression Modeling and Applications. Journal of Statistical Theory and Applications, 19(1), 59-74.

32. Korkmaz, M. C. Yousof, H. M. and Hamedani G. G. (2018a). The exponential Lindley odd log-logistic G family: properties, characterizations and applications. Journal of Statistical Theory and Applications, 17(3), 554 - 571.

33. Korkmaz, M. C., Yousof, H. M., Hamedani G. G. and Ali, M. M. (2018b). The Marshall-Olkin generalized G Poisson family of distributions, Pakistan Journal of Statistics, 34(3), 251-267.

34. Mansour, M. M., Ibrahim, M., Aidi, K., Shafique Butt, N., Ali, M. M., Yousof, H. M. and Hamed, M. S. (2020a). A New Log-Logistic Lifetime Model with Mathematical Properties, Copula, Modified Goodness-of-Fit Test for Validation and Real Data Modeling. Mathematics, 8(9), 1508.

35. Mansour, M. M., Butt, N. S., Ansari, S. I., Yousof, H. M., Ali, M. M. and Ibrahim, M. (2020b). A new exponentiated Weibull distribution's extension: copula, mathematical properties and applications. Contributions to Mathematics, 1 (2020) 57-66.

36. Mansour, M., Korkmaz, M. C., Ali, M. M., Yousof, H. M., Ansari, S. I., \& Ibrahim, M. (2020c). A generalization of the exponentiated Weibull model with properties, Copula and application. Eurasian Bulletin of Mathematics, $3(2), 84-102$.

37. Mansour, M., Rasekhi, M., Ibrahim, M., Aidi, K., Yousof, H. M. and Elrazik, E. A. (2020d). A New Parametric Life Distribution with Modified Bagdonavičius-Nikulin Goodness-of-Fit Test for Censored Validation, Properties, Applications, and Different Estimation Methods. Entropy, 22(5), 592.

38. Mansour, M., Yousof, H. M., Shehata, W. A. and Ibrahim, M. (2020e). A new two parameter Burr XII distribution: properties, copula, different estimation methods and modeling acute bone cancer data. Journal of 
Nonlinear Science and Applications, 13(5), 223-238.

39. Mansour, M. M., Butt, N. S., Yousof, H. M., Ansari, S. I. and Ibrahim, M. (2020f). A Generalization of Reciprocal Exponential Model: Clayton Copula, Statistical Properties and Modeling Skewed and Symmetric Real Data Sets. Pakistan Journal of Statistics and Operation Research, 16(2), 373-386.

40. Merovci, F., Yousof, H. M. and Hamedani, G. G. (2020). The Poisson Topp Leone Generator of Distributions for Lifetime Data: Theory, Characterizations and Applications. Pakistan Journal of Statistics and Operation Research, 16(2), 343-355.

41. Merovci, F., Alizadeh, M., Yousof, H. M. and Hamedani G. G. (2017). The exponentiated transmuted-G family of distributions: theory and applications, Communications in Statistics-Theory and Methods, 46(21), 1080010822 .

42. Nascimento, A. D. C., Silva, K. F., Cordeiro, G. M., Alizadeh, M. and Yousof, H. M. (2019). The odd NadarajahHaghighi family of distributions: properties and applications. Studia Scientiarum Mathematicarum Hungarica, $56(2), 1-26$.

43. Nichols, M.D. and Padgett, W.J. (2006). A bootstrap control chart for Weibull percentiles. Quality and Reliability Engineering International, 22, 141-151.

44. Paranaíba, P. F. P., Ortega, E. M. M., Cordeiro, G. M. and Pescim, R. R. (2011). The beta Burr XII distribution with application to lifetime data. Computation Statistics and Data Analysis, 55, 1118-1136.

45. Rodriguez, R.N. (1977). A guide to the Burr type XII distributions. Biometrika, 64, 129--134.

46. Tadikamalla, P. R. (1980). A look at the Burr and related distributions, International Statistical Review, 48, 337344.

47. Shehata, W. A. M. and Yousof, H. M. (2021). The four-parameter exponentiated Weibull model with Copula, properties and real data modeling. Pakistan Journal of Statistics and Operation Research, 17(3), 649-667.

48. Shehata, W. A. M. and Yousof, H. M. (2021). A novel two-parameter Nadarajah-Haghighi extension: properties, copulas, modeling real data and different estimation methods. Statistics, Optimization \& Information Computing, forthcoming.

49. Shehata, W. A. M., Yousof, H. M. and Aboraya, M. (2021). A Novel Generator of Continuous Probability Distributions for the Asymmetric Left-skewed Bimodal Real-life Data with Properties and Copulas. Pakistan Journal of Statistics and Operation Research, 17(4), 943-961.

50. Yadav, A. S., Goual, H., Alotaibi, R. M., Ali, M. M. and Yousof, H. M. (2020). Validation of the Topp-LeoneLomax model via a modified Nikulin-Rao-Robson goodness-of-fit test with different methods of estimation. Symmetry, 12(1), 57.

51. Yousof, H. M., Alizadeh, M., Jahanshahi, S. M. A., Ramires, T. G., Ghosh, I. and Hamedani, G. G. (2017). The transmuted Topp-Leone $\mathrm{G}$ family of distributions: theory, characterizations and applications. Journal of Data Science, 15(4), 723-740.

52. Yousof, H. M., Altun, E., Ramires, T. G., Alizadeh, M. and Rasekhi, M. (2018). A new family of distributions with properties, regression models and applications, Journal of Statistics and Management Systems, 21, $163-188$.

53. Yousof, H. M., Altun, E., Rasekhi, M., Alizadeh, M., Hamedani, G. G. and Ali, M. M. (2019). A new lifetime model with regression models, characterizations and applications. Communications in Statistics-Simulation and Computation, 48(1), 264-286.

54. Yousof, H. M., Chesneau, C., Hamedani, G. and Ibrahim, M. (2021). A New Discrete Distribution: Properties, Characterizations, Modeling Real Count Data, Bayesian and Non-Bayesian Estimations. Statistica, 81(2), 135162. 